\title{
El estado actual de las vacunas contra las drogas
}

\author{
Maura Epifanía Matus Ortega', Juan Carlos Calva Nieves', Susana Barbosa Méndez', Alberto Salazar Juárez'. \\ I Instituto Nacional de Psiquiatría Ramón de la Fuente Muñiz, México.
}

\section{RESUMEN}

Introducción: por lo común, la adicción a las drogas se trata con psicoterapia y farmacología que evita la unión de las sustancias psicoactivas a receptores específicos en el cerebro. El resultado de estos tratamientos no ha sido del todo satisfactorio, por lo que el desarrollo de terapias más eficaces representa un reto constante para tratar las adicciones. Una alternativa a la farmacología antiadictiva es la vacunación activa dirigida contra las sustancias de abuso. Objetivo: esta revisión reúne la información disponible sobre los fundamentos y avances científicos en la generación de una terapia inmunológica, que coadyuve al tratamiento de la adicción a sustancias como la heroína-morfina, la cocaína, la nicotina y la anfetamina. Método: se consideraron los reportes científicos disponibles en PubMed -de 2005 a abril de 2017-, sobre los fundamentos, la metodología empleada, los estudios preclínicos y clínicos, y los resultados obtenidos en dichas investigaciones para generar vacunas contra las drogas. Resultados: las vacunas lograron mitigar los efectos producidos por las sustancias en los estudios preclínicos en modelos de estudio en animales; sin embargo, con pacientes humanos los resultados no han sido del todo satisfactorios. Discusión y conclusiones: a pesar de los esfuerzos realizados por diferentes grupos de investigación y compañías farmacéuticas para generar vacunas terapéuticas contra el uso de diferentes drogas, ninguna ha alcanzado la fase III de estudios clínicos. En la actualidad, se continúa con los esfuerzos para lograr que las vacunas contra las adicciones alcancen su máxima eficiencia y eficacia, y contribuyan al tratamiento de la adicción a las drogas.

Palabras clave: vacunas, morfina, heroína, nicotina, metanfetamina.

\begin{abstract}
Introduction: traditional approach for drug addiction includes treatment with psychological assistance alone or in combination with pharmacotherapy aimed at blocking the binding of addictive drugs to their specific brain receptors. Success rate of these traditional treatments is not satisfactory; hence, alternative approaches should be explored, including immunotherapy targeting drugs of abuse. Objective: this paper focuses in a review of available literature concerning immunotherapy for the following drugs of abuse: morphine, cocaine, nicotine and methamphetamine, in order to be used as part of addiction treatment. Method: a search was conducted for scientific reports in PubMed from January, 2005 to April, 2017, focused on the scientific principles, method, preclinical and clinical trials and their results on the field of vaccination against substance of abuse. Results: the available vaccines against substance abuse succeed in blocking the drug's reinforcing effects and mitigate drug-induced behaviors in preclinical trials in animal models. On the contrary, clinical trials in humans did not show therapeutic success. Discussion and conclusions: both academic research groups and pharmaceutical companies have published a number of pre-clinical research works on the field of vaccines for substance abuse, but thus far pre-clinical findings are not supported by results of the clinical trials. At present, efforts persist to improve vaccine efficacy and efficiency in humans for developing immunotherapy as a viable option for patients seeking treatment for drug addiction.
\end{abstract}

Key words: vaccines, morphine, heroin, nicotine, methamphetamine.

\footnotetext{
Autora de correspondencia:

Maura Epifanía Matus Ortega. Instituto Nacional de Psiquiatría Ramón de la Fuente Muñiz. Calzada México-Xochimilco núm. 101, Delegación Tlalpan, C.P. 14370, Ciudad de México. Correo electrónico: mematuso@gmail.com

Recibido: 19 de julio de 2017.

Aceptado: 25 de septiembre de 2017.

DOI: 10.28931/riiad.2017.2.05
} 


\section{INTRODUCCIÓN}

Por lo común, el abuso de drogas se trata con psicoterapia y se combina con tratamientos farmacológicos que consisten en una estrategia de substitución de la droga por un compuesto químico análogo con actividad farmacológica similar, pero con propiedades farmacocinéticas diferentes que bloquean la unión de la droga con receptores específicos en el cerebro. El mejor ejemplo de esta estrategia es el empleo de agonistas, como metadona y buprenorfina, y antagonistas, como naloxona y naltrexona, para la adicción a los opiáceos.

Para los dependientes de la nicotina, la alternativa consiste en administrar la sustancia en diferentes formas farmacéuticas (parches, aerosoles, gomas, etcétera), y compuestos con actividad de agonistas/antagonistas a nivel del receptor, como la vareniclina, o inhibidores de la recaptura de neurotransmisores, como el buprobión; cabe señalar que esta farmacología conlleva efectos colaterales diversos (Tabla 1).

La severidad de los síntomas del síndrome de abstinencia es de los mayores retos en el tratamiento de las adicciones y se ha reportado que la mirtazapina reduce la causada por el consumo de morfina, cocaína y metanfetamina, en animales y humanos (Salazar-Juárez et al., 2016). Así, la búsqueda de terapias altamente efectivas y eficientes representa un reto para el tratamiento de las adicciones. Una alternativa a la farmacología tradicional es la inmunoterapia o vacunación dirigida a sustancias de abuso (Brimijoin, Shen, Orson, \& Kosten, 2013; Kinsey, 2014; Zalewska-Kaszubska, 2015).

La investigación en inmunoterapia para las drogas considera que una vacuna estimula al sistema inmune al identificar a las moléculas adictivas y a sus metabolitos como antígenos, para producir inmunoglobulinas específicas neutralizantes. Por lo general, las drogas son pequeñas moléculas lipofilicas que no despiertan la respuesta inmune por sí mismas, de manera que se modifican y conjugan químicamente con una proteína portadora compleja para activar la respuesta inmune humoral y generar anticuerpos específicos. Las proteínas portadoras más usadas son la albúmina sérica bovina (BSA, del inglés, bovine serum albumen), la KLH (del inglés, Keyhole limpet haemocyanin) y el toxoide tetánico (TT), entre otras. La unión de los anticuerpos específicos a la droga evita que ésta cruce la barrera hematoencefálica, lo que detiene los procesos neurofarmacológicos de la droga y rompe el ciclo de recompensa-adicción, como se esquematiza en la Figura 1 (Kinsey, Jackson, \& Orson, 2009; Kosten \& Owens, 2005; Montoya, 2012; Moreno \& Janda, 2009; Shen, Orson, \& Kosten, 2011).

\section{MÉTODO}

Siguiendo los criterios PRISMA (Moher, Liberati, Tetzlaff, \& Altman, 2009), se realizó una búsqueda de la literatura

Tabla 1

Fármacos más comunes para el tratamiento de las adicciones y los efectos colaterales relevantes que promueven recaídas y altas tasas de abandono de los tratamientos

\begin{tabular}{|c|c|c|}
\hline Adicción & Farmacoterapia & Efectos colaterales más frecuentes \\
\hline \multirow[t]{5}{*}{ Opioides } & Metadona & Constipación, náusea, depresión respiratoria, prurito. \\
\hline & Levacetilmetadol & $\begin{array}{l}\text { Gastrointestinales (dolor abdominal, diarrea, sequedad de boca y estre- } \\
\text { ñimiento), respiratorias (tos y rinitis), dermatológicas (exantema), uroge- } \\
\text { nitales (disminución de la libido y retraso en la eyaculación), bradicardia, } \\
\text { artralgia, visión borrosa, astenia y síntomas gripales. }\end{array}$ \\
\hline & Buprenorfina & Sedación, constipación, síndrome de abstinencia e insomnio. \\
\hline & Naloxona & $\begin{array}{l}\text { Náusea y vómito; ocasionalmente hipertensión severa, edema pulmonar, } \\
\text { taquicardia y arritmias. }\end{array}$ \\
\hline & Naltrexona & $\begin{array}{l}\text { Insomnio, dolor de cabeza, ansiedad, náusea y vómito, cólico intestinal, } \\
\text { fatiga, dolor muscular y articular. }\end{array}$ \\
\hline \multirow[t]{3}{*}{ Nicotina } & Nicotina de reemplazo & $\begin{array}{l}\text { Alteración del sueño, hipo, acidez, insensibilidad oral (goma), irritación en } \\
\text { boca y garganta (inhalador). }\end{array}$ \\
\hline & Buprobión & Resequedad oral, insomnio. \\
\hline & Vareniclina & Náusea o vómito, alteración de sueño y flatulencias. \\
\hline
\end{tabular}

Cocaína, anfetamina y Ninguna otros estimulantes

Fuente: Chou et al, 2014; Barnett, Twycross, Mihalyo, \& Wilcock, 2014; Davids \& Gatspar, 2004; Hatsukami, Stead, \& Gupta, 2008; Veilleux, Colvin, Anderson, York, \& Heinz, 2010. 


\section{Sistema nervioso central}

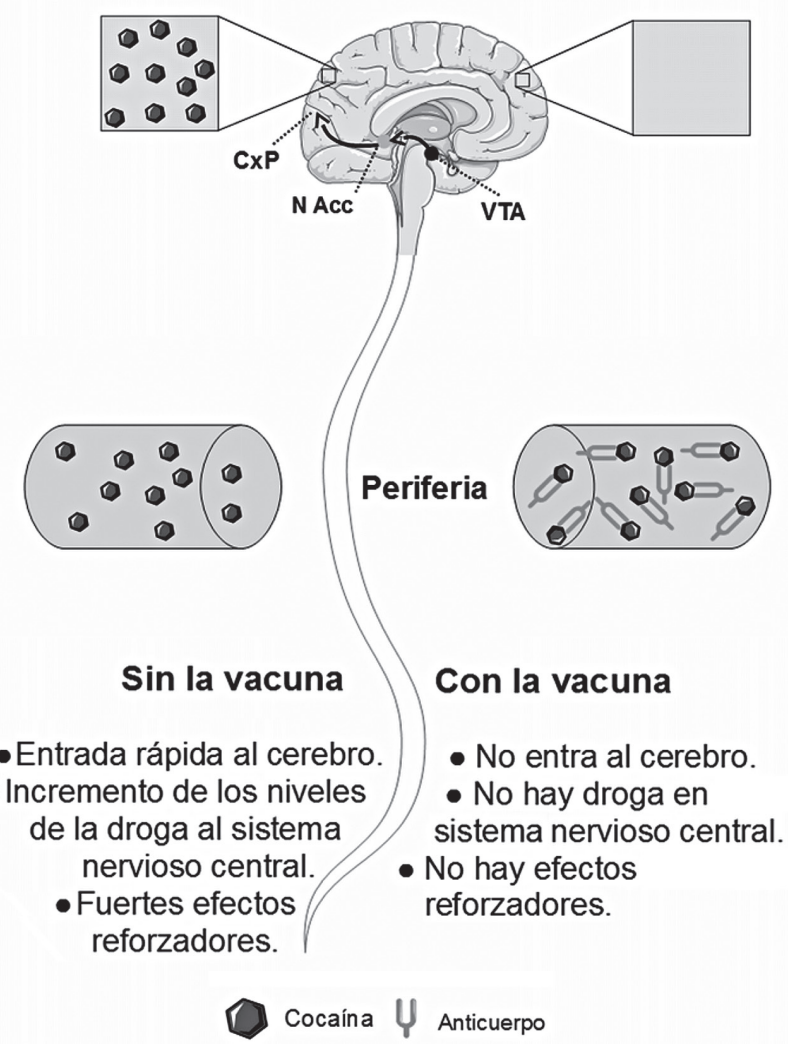

Figura 1. Una vacuna terapéutica genera altos títulos de anticuerpos específicos que se unen a la droga blanco en la periferia, con lo que se produce un complejo antígeno-anticuerpo, que no puede atravesar la barrera hematoecefálica; de esta manera se impide la penetración de la droga al Sistema Nervioso Central eliminando la acción reforzadora de la droga sobre los circuitos mesocorticolímbicos de recompensa. Abreviaturas: CxP: Corteza prefrontal; NAcc: núcleo accumbens; VTA: área tegmental ventral. Modificado de Ohia-Nwoko, Kosten, \& Haile, 2016.

científica publicada en PubMed entre el 1 de enero de 2005 y el 30 de abril de 2017 (fecha en la que se inició esta revisión). Las palabras clave usadas para la búsqueda sistemática fueron vaccine AND addiction, con las que se obtuvieron 190 artículos; de este grupo se seleccionaron 98 enfocados en opioides (morfina, heroína), metanfetaminas, cocaína y nicotina, y sus artículos de revisión; como criterio de exclusión se consideraron los textos que trataban exclusivamente de oxicodona y anticuerpos monoclonales y los que estaban en idiomas diferentes al inglés y el español (92 artículos). Posteriormente, se eligieron 54 artículos y se excluyeron 44 más a los que no se tuvo un completo acceso (Figura 2).

\section{RESULTADOS}

\section{Vacunas para morfina y heroína}

La búsqueda de vacunas para las sustancias de abuso inició con el grupo de los opioides en los años setenta del siglo pasado. En esa década, Spector y Parker desarrollaron una vacuna para morfina y generaron anticuerpos en conejos para identificar morfina en radioinmunoensayos. Más tarde, Ryan y su grupo de investigación reportaron el hallazgo de anticuerpos contra morfina en sueros de usuarios de heroína; por su parte, el grupo de investigación de Hill inmunizó conejos contra esta sustancia empleando la molécula 6-succinilmorfina conjugada con BSA (Kinsey, 2014).

Los grupos de investigación de Wainer y Bonese desarrollaron vacunas para morfina y heroína con la capacidad de unirse a estas sustancias y retenerlas en el suero; demostraron que la conducta de autoadministración de heroína se redujo de manera significativa en primates no humanos vacunados. Más tarde, los grupos de investigación de Bonese, Killian, Raleig y Stowe desarrollaron estudios clínicos en roedores y primates, en los que la administración de la vacuna contra morfina redujo los efectos conductuales originados por el uso de heroína (Zalewska-Kaszubska, 2015).

El interés en el desarrollo de vacunas para las drogas tuvo su auge a partir de la década de los noventa del siglo pasado. El grupo de investigación de Akbarzadeh

\section{Identificación}

\section{0 artículos identificados en PubMed}

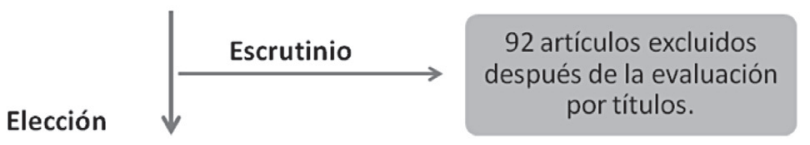

98 artículos seleccionados

después de la evaluación portítulos.

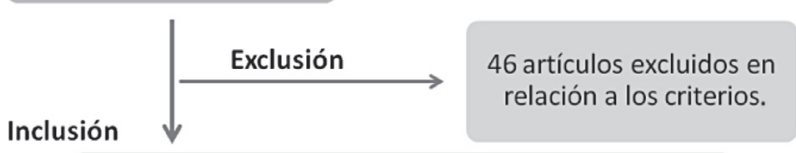

\section{2 artículos incluidos}

Figura 2. Diagrama de flujo de la búsqueda de literatura científica de acuerdo con los criterios PRISMA para la identificación e inclusión de estudios en la presente revisión. Los criterios de inclusión y exclusión se describen en el método. 
sintetizó una vacuna para morfina conjugada con BSA, la M6S-BSA (morphine-6-succinyl-bovine serum albumin), que se probó en ratones (Akbarzadeh, Mehraby, Zarbakhsh, \& Farzaneh, 1999) y después fue aplicada en estudios clínicos con 347 personas adictas, en 2009, y 436, en 2012.

Los participantes en la investigación generaron altos títulos de anticuerpos después de 30 días de la primera inmunización, que permanecieron altos hasta un año después; la vacuna fue bien tolerada y sin efectos adversos considerables (Akbarzadeh et al., 2009; Farhangi et al., 2012).

Torres y su grupo optimizaron el procedimiento de acoplamiento para la síntesis de un hapteno del tipo morfina-heroína con BSA, con el que obtuvieron altos rendimientos y buena reproducibilidad; concluyeron que este procedimiento se puede aplicar para el diseño de diferentes haptenos y proteínas portadoras (Torres et al., 2014). El uso clínico de BSA no está permitido en todas partes del mundo, por lo que su empleo se suspendió en el desarrollo de vacunas para humanos.

En 2006, Antón y Leff generaron una vacuna bivalente para morfina y heroína, utilizando como hapteno a la morfina-6-hemisuccinato unida a TT, proteína portadora altamente inmunogénica; se obtuvieron altos niveles de anticuerpos con capacidad de reconocer tanto a la heroína como a la morfina. Al administrarse en ratas adictas, los anticuerpos generados por esta vacuna redujeron la conducta de autoadministración de heroína y morfina (Anton \& Leff, 2006).

Por su parte, Li y colaboradores emplearon una vacuna conjugada en la que se usó como hapteno a la 6-glutarilmorfina unida a KLH, que atenuó la conducta y los efectos psicoactivos de la heroína en ratas; además, se incrementó la concentración de anticuerpos específicos para morfina y heroína que no tuvieron especificidad de reconocimiento por otras sustancias opioides, tales como buprenorfina, metadona, naloxona, naltrexona, nalorfina o codeína (Li et al., 2011; Li et al., 2015). Esta propiedad es relevante debido a que estos opioides pueden usarse como terapia farmacológica complementaria a la adicción y se usan de manera amplia como analgésicos.

Kosten y su grupo reportaron resultados prometedores con una vacuna KLH-6-SM, en la que los niveles de morfina en ratas vacunadas disminuyeron 26 veces más, respecto a los controles (Kosten et al., 2013).

Raleigh y su grupo evaluaron el efecto de una vacuna de morfina conjugada con KLH en la adquisición, el mantenimiento y la reinstalación de la autoadministración de heroína en ratas; de su evaluación concluyeron que la inoculación bloqueó la reinstalación de la conducta de autoadministración de dosis altas de heroína (0.6 mg/kg, s. c., dosis que está dentro del rango que en humanos producen recompensa), redujo la potencia de la droga y disminuyó las concentraciones de heroína, sus metabolitos activos y morfina en cerebro, dado que estas moléculas fueron retenidas en el plasma (Raleigh, Pentel, \& LeSage, 2014).

Otro desarrollo importante es el de una vacuna "dinámica”, con la capacidad de generar anticuerpos contra la heroína y sus metabolitos psicoactivos debido a la presentación de una estructura de hapteno múltiple al sistema inmune. La administración de esta vacuna a ratas dependientes de heroína previno el efecto de recompensa, la conducta de búsqueda de la droga y la reinstalación de la conducta de autoadministración compulsiva después de un periodo de abstinencia (Schlosburg et al., 2013; Stowe, Schlosburg et al., 2011; Stowe, Vendruscolo et al., 2011).

El grupo de Janda continúa mejorando la formulación de esta vacuna y en 2014 evaluaron el empleo de un agonista del TLR9 como adyuvante, con el fin de mejorar la respuesta inmune; la eficacia de este adyuvante, CpG ODN 1829, ha sido probada en estudios de fase clínica I y II: es estable, su empleo es barato, es fácil de obtener y de manipular para la formulación de vacunas. Con esto se obtuvieron mayores títulos de anticuerpos (cuatro veces más de lgG1), con mayor afinidad de unión a los opioides evaluados (27 veces para heroína, nueve veces para $6 \mathrm{AM}$ y 12 veces para morfina). El efecto protector de la formulación se incrementó de 10 a 13 veces respecto a los grupos sin vacunar y de dos a siete veces en los vacunados sin el adyuvante (Bremer, Schlosburg, Lively, \& Janda, 2014). Estos resultados vuelven a la vacuna muy prometedora para apoyar el tratamiento de la adicción.

\section{Vacunas para cocaína}

En la actualidad no existe una terapia farmacológica efectiva para la adicción a la cocaína, de manera que la inmunoterapia representa una oportunidad para su desarrollo (Orson et al., 2014).

Kantak y colaboradores desarrollaron una vacuna en la que se conjuga la molécula de succinil norcocaína con la toxina B del cólera; ésta bloqueó de manera efectiva la autoadministración de cocaína en ratas vacunadas debido a que generaron altos títulos de anticuerpos específicos contra cocaína, previniendo que la sustancia entrara al cerebro (Shorter \& Kosten, 2011). En un inicio, esta vacuna se llamó IPC-1010 y fue licenciada en exclusiva a la compañía Immu-Logic Pharmaceutical Corp., Waltman, Mass., USA (Zalewska-Kaszubska, 2015). Después, ICP-1010 fue renombrada como TA$C D$, cuando la propiedad fue transferida a la compañía Xenova (Cantab). El primer estudio clínico con TA-CD 
evaluó la inmunogenicidad y la seguridad de la vacuna, que mostró buena tolerancia; no se reportaron efectos adversos (Martell, Mitchell, Poling, Gonsai, \& Kosten, 2005; Martell et al., 2009) y se generaron anticuerpos desde la segunda administración; los mayores títulos se obtuvieron después de tres meses y declinaron a niveles basales después de un año.

El siguiente estudio clínico abierto mostró que las personas vacunadas volvieron a consumir cocaína pero sin experimentar su acción psicoestimulante (Martell et al, 2005). La fase Ilb del estudio clínico involucró a 115 pacientes ambulatorios con adicción a opioides y cocaína en tratamiento de sustitución con metadona. Los niveles de anticuerpos alcanzados después de la vacunación difirieron de manera significativa entre los participantes del estudio. Se requirió alcanzar altos niveles de $\operatorname{lgG}(\geq 43 \mu \mathrm{g} / \mathrm{ml})$ para reducir significativamente el acceso de la sustancia al cerebro y prevenir sus efectos eufóricos, condición que se obtuvo en sólo $38 \%$ de los pacientes vacunados (Martell et al., 2009).

Debido a que en la mayoría de los sujetos, los niveles de IgG generados no fueron satisfactorios, se midió el nivel de anticuerpos IgM para cocaína. Se encontró que en los pacientes había anticuerpos IgM desde antes de la vacunación, lo que se explicó como resultado del uso repetido de cocaína. Los autores señalaron que esto fue un impedimento para generar altos niveles de anticuerpos IgG en respuesta a la vacuna; por lo que los participantes no eran candidatos adecuados para el tratamiento (Orson et al., 2013). Para solucionar este problema, se realizó un segundo análisis de pacientes dependientes, a fin de identificar la relación entre el polimorfismo del gen de dopamina $\beta$-hidroxilasa ( $\mathrm{DBH}$, enzima que convierte a la dopamina en noradrenalina) y la efectividad de la vacuna para cocaína (Martell et al., 2009). Se observó que los pacientes con baja actividad de DBH (genotipos rs 1611115) redujeron de forma significativa la ingesta de cocaína, en comparación con el grupo control, después de recibir la vacuna. En el grupo placebo no hubo diferencia entre los pacientes con el genotipo rs1611115 y el resto de los pacientes. Los autores concluyeron que la identificación del genotipo DBH en los pacientes adictos a cocaína puede servir para detectar a aquellas personas para quienes la vacuna no es efectiva. En la fase III del estudio clínico, los pacientes vacunados no redujeron la administración de cocaína, a pesar de haber alcanzado los niveles apropiados de anticuerpos (Kosten et al., 2014).

Se han desarrollado vacunas a partir de vectores adenovirales, como la dAd5GNC y la dAd5GNE, que en ratones generaron títulos de anticuerpos suficientes para eliminar la estimulación psicomotora inducida por la droga (Hicks et al., 2011; Wee et al., 2012). Después de varias administraciones de dAd5GNE, se generaron altos títulos de anticuerpos y se redujo de 62 a menos de $20 \%$ la unión de cocaína al transportador de dopamina en primates no humanos, con lo cual se superó el límite que permite el efecto psicoestimulante de la cocaína (47\%; Maoz et al., 2013).

Haney y colaboradores investigaron el efecto protector de la vacuna ante una sobredosis de cocaína en voluntarios dependientes. A éstos se les administró diariamente una dosis de 25 y $50 \mathrm{mg}$ de cocaína. En individuos que produjeron títulos de anticuerpos significativos se observó una reducción importante del efecto psicoestimulante, pero al mismo tiempo, con dosis altas, se incrementó el ritmo cardiaco. En apariencia existe un riesgo serio de que estos pacientes traten de incrementar la administración de cocaína para superar el efecto de la vacuna, por lo que estas terapias deben tomarse con mucha precaución (Haney, Gunderson, Jiang, Collins, \& Foltin, 2010).

\section{Vacunas para nicotina}

Pfizer ha trabajado de forma sistemática en extensos programas para optimizar las vacunas para nicotina y como resultado de ello se tienen las vacunas análogas NicQB y NicVax. El efecto de la Nic-Vax (3'-AmNic-rEPA) se estudió en siete personas que fumaron un promedio de 19 cigarros al día, durante al menos 10 años. La vacunación disminuyó la unión de la nicotina en $12.5 \%$, así como la ansiedad y el número de cigarros fumados por día (Esterlis et al., 2013). Dos ensayos clínicos en fase I evaluaron la seguridad e inmunogenicidad de la NicVax (Hatsukami et al., 2005; Wagena, de Vos, Horwith, \& van Schayck, 2008) y demostraron que es segura, bien tolerada y no provoca efectos adversos serios. El estudio de fase II, que incluyó a 301 fumadores, investigó la relación entre el grado de abstinencia y la concentración de anticuerpos en el plasma sanguíneo de los sujetos (Hatsukami et al., 2011). Se encontró que 16\% de los pacientes con altos títulos de anticuerpos, aún después de un año de la última vacunación, permaneció en abstinencia, mientras que sólo lo logró $6 \%$ del grupo placebo. En contraste, los pacientes con altos títulos de anticuerpos que no dejaron de fumar disminuyeron su consumo de cigarros a cinco por semana (Hatsukami et al., 2005).

La vacuna NicVax es la única que ha alcanzado la fase III de los ensayos clínicos. En el estudio conducido por Nabi, que incluyó cerca de 1000 personas que fumaban al menos 10 cigarros por día, no hubo diferencias con el grupo placebo (Fahim, Kessler, \& Kalnik, 2013; Zalewska-Kaszubska, 2015), tampoco incrementó la abstinencia en fumadores que fueron tratados al mismo tiempo con vareniclina (Hoogsteder, Kotz, van Spiegel, Vie- 
chtbauer, \& van Schayck, 2014). Más aún, Havermans y colaboradores mostraron efectos no significativos en la actividad cerebral en respuesta a la nicotina; por lo tanto, concluyeron que esta vacuna no es efectiva en la cesación de fumar, probablemente debido a su incapacidad de generar niveles terapéuticos de anticuerpos específicos contra nicotina (Havermans, Vuurman, van den Hurk, Hoogsteder, \& van Schayck, 2014).

$\mathrm{Nic} Q b$ se desarrolló con el hapteno de nicotina conjugado a una VLP (partícula parecida a virus, virus-like particle) formada por una proteína de cápside del bacteriófago Qb. En estudios preclínicos se observó que ratones inmunizados tuvieron concentraciones promedio de nicotina en sus cerebros de $57 \%$ por debajo del grupo control. La seguridad de la vacuna NicQb se evaluó en estudios de fase clínica I en 32 voluntarios sanos no fumadores; mostró ser segura y bien tolerada; sus efectos adversos principales fueron reacciones locales (Maurer et al., 2005). Los estudios clínicos de fase II confirmaron la seguridad de NicQb; su alta inmunogenicidad se observó en todos los sujetos desde la primera inyección (Cornuz et al., 2008). Al cabo de dos meses, los individuos tratados con NicQb mostraron una abstinencia más alta (47\%), respecto al grupo placebo (35.1\%); sin embargo, después, entre los dos y seis meses, no se observaron diferencias. Cuando se analizó la población de participantes, se observó que los sujetos con las concentraciones más altas de anticuerpos tuvieron una abstinencia mayor durante los meses dos a seis (56.6\%), que los individuos tratados con placebo (31.3\%). Incluso después de 12 meses de observación, la diferencia en la abstinencia entre los sujetos del grupo placebo y los que tuvieron los más altos títulos de anticuerpos permaneció en 20.2\% (Cornuz et al., 2008).

La vacuna Niccine mostró buena tolerancia en ensayos clínicos preliminares, no obstante, los estudios de fase clínica II revelaron que la abstinencia en el grupo vacunado no difirió del grupo placebo y, dada la pérdida de eficacia de la vacuna, la fábrica tuvo que suspender su desarrollo futuro (Tonstad et al., 2013).

\section{Segunda generación de vacunas para nicotina}

Los resultados desalentadores de los estudios clínicos de primera generación de vacunas promovieron que se modificaran y evaluaran nuevas formulaciones.

Pfizer y Selecta Biosciences iniciaron el desarrollo de la segunda generación de vacunas para nicotina, mismas que se han incluido en estudios clínicos iniciales. Pfizer adquirió Coley Pharmaceutical y accedió al programa del potente agonista de TLR (del inglés, Toll-like receptor), que permitió el desarrollo de la segunda gene- ración de vacunas contra nicotina: la NIC7-001, que se desarrolló tomando como referencia a NicVac y NicQB. La NIC7-001 está conformada por el hapteno de nicotina conjugado a CRM (cross-reacting material of diphtheria toxin) y generó altos títulos de anticuerpos específicos y de alta afinidad, lo que permitió una reducción significativa de los niveles de nicotina en estudios preclínicos en cerebros de ratones y primates no humanos; asimismo, logró inducir niveles de IgG de alta afinidad en 60\% o más de la población evaluada.

La nanovacuna sintética SEL-068 de Selecta Biosciencies induce la producción de títulos de anticuerpos de alta afinidad antinicotina tanto en ratones como en primates no humanos (Fraser et al., 2014); previene de manera efectiva la expresión de los efectos discriminativos generados por la nicotina en primates naïve a nicotina y produce una reducción sustantiva (de casi seis veces) y de largo plazo ( $\geq 25$ semanas después de la vacunación) en la potencia a la cual la nicotina produce efectos discriminables en primates no humanos (Desai \& Bergman, 2015). En apariencia, las nanovacunas para nicotina pueden ser útiles como medida de prevención en individuos no expuestos o como forma de reducir el riesgo de readquisición durante la abstinencia a largo plazo en antiguos fumadores (Desai \& Bergman, 2016). En noviembre de 2011, se inició un estudio de fase clínica I cuyo propósito fue el de evaluar la seguridad, la tolerancia y el perfil farmacodinámico de la vacuna en voluntarios sanos fumadores y no fumadores. La vacuna fue bien tolerada y logró generar una adecuada cantidad de anticuerpos, la compañía considera necesario hacer cambios en su formulación para incrementar la potencia de la respuesta inmune en humanos, lo que genera un retraso importante, de varios años, para llevar a cabo los estudios clínicos de fase II (Montoya, 2016).

\section{Vacunas para metanfetaminas}

Se han evaluado diferentes vacunas antimetanfetamina en modelos de ratones, la mayoría usan como proteína portadora a la KLH, pero difieren en el diseño del hapteno y los adyuvantes.

La SMA-KLH tiene como hapteno a la succinil metanfetamina (SMA, del inglés, succinyl methamphetamine), unida a KLH. Esta vacuna se administró con el lípido monofosfolil A, como adyuvante, y generó anticuerpos que bloquearon los efectos en la conducta de preferencia de lugar y la actividad locomotora producidos por la metanfetamina en ratones vacunados. Se generaron anticuerpos después de la segunda semana de vacunación, que mostraron un pico a partir de las seis semanas y permanecieron elevados hasta la semana treinta y cinco (Shen 
et al., 2013). Se evaluó también un modelo con el mismo hapteno SMA usando TT como proteína portadora; para la vacuna SMA-TT, se utilizó aluminio como adyuvante y se evaluó el uso de un segundo adyuvante, el E6020 (TRL4) o el entolimod (TRL5), un adyuvante derivado de la flagelina de salmonela y un agonista de TLR5, respectivamente; éstos han mostrado eficacia médica previa en modelos de estudio en animales. Con esta vacuna se evaluó la actividad locomotora y conductual de preferencia de lugar y se reportó una reducción de los efectos condicionados y la actividad locomotora en respuesta a la administración de metanfetamina, pero no disminuyó la conducta de preferencia de lugar. Por otro lado, reportaron que entre 3 y $4 \%$ de los ratones no produjo anticuerpos para metanfetamina, aún cuando se incluían los adyuvantes E6020 y entolimod (Montoya, 2016).

El grupo de investigación de Duryee diseñó una vacuna empleando un adyuvante peptídico, el EP54 (SFKPMPLaR), que es agonista de C5a65-74 del complemento y el epítope de células T de la toxina tetánica unida covalentemente a EP54; como hapteno se usó la metanfetamina. La administración de la vacuna en ratas generó anticuerpos específicos con capacidad de modificar la conducta de autoadministración, incrementándola. Los autores sugieren que esta modificación en la conducta se debe a la necesidad de los sujetos de administrarse metanfetamina, porque la consumida de manera previa se encuentra unida a los anticuerpos y no llega al cerebro (Duryee et al., 2009).

En 2012, Moreno y su grupo diseñaron varios haptenos para metanfetamina con estructura química estable mediante modelaje molecular. Una de las vacunas diseñadas, la MH6, bloqueó, en modelo de ratas, el efecto de reducción de temperatura y la actividad locomotora inducidas por metanfetamina; la vacuna protegió a las ratas de los efectos fisiológicos y conductuales de la metanfetamina y atenuó la conducta de la autoadministración de la droga (Miller et al., 2013; Miller et al., 2015; Moreno, Mayorov, \& Janda, 2011).

La vacuna $I_{\mathrm{KLH}_{\mathrm{H}}} \mathrm{SMO9}$, en la que se conjugó el hapteno HSMO9 -similar a la metanfetamina con inmunocianina (proteína derivada de KLH)-, se evaluó mediante la inmunización repetida con altas dosis y no produjo efectos adversos en la salud, peso corporal o desempeño durante las pruebas de conducta de mantenimiento de alimento (Rüedi-Bettschen et al., 2013). Las ratas inmunizadas con $\mathrm{IC}_{\mathrm{KLH}}-\mathrm{SMO}$ tuvieron concentraciones séricas de metanfetamina aún mayores para todas las dosis, indicando que los anticuerpos generados secuestraron a la metanfetamina en el torrente sanguíneo. Los resultados sugieren que la vacuna puede mejorar la salud de los pacientes aún cuando se administren dosis altas de metanfetamina.

\section{DISCUSIÓN Y CONCLUSIONES}

Las vacunas contra las drogas continúan representando una alternativa importante para el tratamiento de las adicciones, en virtud de sus escasos efectos colaterales. Al no permitir que las drogas lleguen al Sistema Nervioso Central pueden prevenir el daño cerebral y los efectos negativos subsecuentes.

Contrario a la farmacoterapia actual de uso clínico, las vacunas tienen un efecto terapéutico prolongado al generar altos niveles de anticuerpos específicos por periodos largos, de 30 días a un año; además, disminuyen los problemas de desapego al tratamiento de los pacientes y no interfieren con la bioquímica cerebral de los centros placenteros endógenos naturales. Los estudios preclínicos han mostrado que la mayoría de las vacunas en desarrollo reducen la actividad motora, la conducta de preferencia de lugar y la autoadministración de las drogas; desafortunadamente, estos beneficios terapéuticos no se han reproducido en estudios de fases clínicas y ninguna de las vacunas ha logrado superar la fase III de estudios clínicos.

Los resultados de los últimos estudios clínicos de las vacunas para cocaína y nicotina han sido desalentadores, generaron bajos títulos de anticuerpos y su aplicación no logró que los pacientes controlaran la adicción. Por lo regular, sólo un tercio de las personas vacunadas logra producir la concentración de anticuerpos suficientes para evitar la entrada de las sustancias al cerebro, por lo que es necesario complementar el tratamiento con terapia farmacológica para reducir el síndrome de abstinencia que experimentarán los pacientes y evitar una sobredosificación accidental.

Los grupos de investigación continúan trabajando para optimizar las vacunas contra las distintas adicciones; además de estar enfocados en mejorar la cantidad de los anticuerpos producidos, ahora consideran la variabilidad de la respuesta inmune de los individuos candidatos a ser vacunados. En este sentido, se vuelve indispensable desarrollar y disponer de estudios farmacogenómicos individuales y poblacionales que permitan seleccionar a los candidatos óptimos al tratamiento con vacunas.

Se debe entender que si bien las vacunas no son un tratamiento universal para todas las complicaciones que provoca la adicción, sí son una valiosa herramienta terapéutica para su tratamiento si se elige de manera adecuada a los candidatos; para quienes las vacunas no sean la mejor opción, pueden ser un complemento de apoyo en combinación con terapia y rehabilitación.

\section{FUENTES DE FINANCIAMIENTO}

La presente investigación fue financiada por la Fundación Gonzalo Río Arronte, IAP. Las opiniones, hallazgos 
y conclusiones aquí presentadas son las de los autores y no necesariamente reflejan las del patrocinador.

\section{CONFLICTOS DE INTERÉS}

Declaramos que no existe conflicto de interés por parte de los autores.

\section{AGRADECIMIENTOS}

Los autores extienden un especial agradecimiento al QFB José Joel Alejandro Mellado Balderas por el apoyo en la corrección de estilo del resumen en inglés del presente manuscrito.

\section{REFERENCIAS}

Akbarzadeh, A., Mehraby, M., Zarbakhsh, M., \& Farzaneh, H. (1999). Design and synthesis of a morphine-6-succinyl-bovine serum albumin hapten for vaccine development. Biotechnology Applied Biochemistry, 30(2), 139-146. doi: 10.1111/j.1470-8744.1999. tb00904.x

Akbarzadeh, A., Norouzian, D., Farhangi, A., Mehrabi, M., Chiani, M., Zare, D., ... Nikdel, A. (2009). Immunotherapy of 347 volunteer outpatient morphine addicts by human therapeutic morphine vaccine in Kermanshah Province of Iran. Journal Pharmacology and Toxicology, 4(1), 30-35. doi: 10.3923/jpt.2009.30.35

Anton, B., \& Leff, P. (2006). A novel bivalent morphine/heroin vaccine that prevents relapse to heroin addiction in rodents. Vaccine 24(16), 3232-3240. doi: 10.1016/j.vaccine.2006.01.047

Barnett, V., Twycross, R., Mihalyo, M., \& Wilcock, A. (2014). Opioid antagonists. Journal of Pain and Symptom Managemen, 47(2), 341-352. doi: 10.1016/j.jpainsymman.2013.12.223

Bremer, P. T., Schlosburg, J. E., Lively, J. M., \& Janda, K. D. (2014). Injection route and TLR9 agonist addition significantly impact heroin vaccine efficacy. Molecular Pharmaceutics, 11(3), 10751080. doi: $10.1021 / \mathrm{mp} 400631 \mathrm{w}$

Brimijoin, S., Shen, X., Orson, F., \& Kosten, T. (2013). Prospects, promise and problems on the road to effective vaccines and related therapies for substance abuse. Expert Review of Vaccines, 12(3), 323-332. doi: 10.1586/erv.13.1

Chou, R., Cruciani, R. A., Fiellin, D. A., Compton, P., Farrar, J. T., Haigney, M. C., ... Zeltzer, L. (2014). Methadone safety: a clinical practice guideline from the American Pain Society and college on problems of drug dependence, in collaboration with the Heart Rhythm Society. The Journal of Pain, 15(4), 321-337. doi: 10.1016/j.jpain.2014.01.494

Cornuz, J., Zwahlen, S., Jungi, W. F., Osterwalder, J., Klingler, K., van Melle, G., ... Cerny, T. (2008). A vaccine against nicotine for smoking cessation: a randomized controlled trial. PLOS ONE, 3(6), e2547. doi: 10.1371/journal.pone.0002547

Davids, E., \& Gastpar, M. (2004). Buprenorphine in the treatment of opioid dependence. European Neuropsychopharmacology,
14(3), 209-216. doi:10.1016/S0924-977X(03)00146-9

Desai, R. I., \& Bergman, J. (2015). Effects of the nanoparticle-based vaccine, SEL-068, on nicotine discrimination in squirrel monkeys. Neuropsychopharmacology, 40(9), 2207-2216. doi: 10.1038/npp.2015.64

Desai, R. I., \& Bergman, J. (2016). Nicotine-targeting nano-vaccines for smoking cessation. Neuropsychopharmacology, 41(1), 377378. doi: 10.1038/npp.2015.234

Duryee, M. J., Bevins, R. A., Reichel, C. M., Murray, J. E., Dong, Y., Thiele, G. M., ... Sanderson, S. D. (2009). Immune responses to methamphetamine by active immunization with peptide-based, molecular adjuvant-containing vaccines. Vaccine, 27(22), 29812988. doi: 10.1016/j.vaccine.2009.02.105

Esterlis, I., Hannestad, J. O., Perkins, E., Bois, F., D'Souza, D. C., Tyndale, R. F., ... O'Malley, S. S. (2013). Effect of a nicotine vaccine on nicotine binding to $\mathrm{b}^{*}$-nicotinic acetylcholine receptors in vivo in human tobacco smokers. American Journal of Psychiatry, 170(4), 399-407. doi: 10.1176/appi.ajp.2012.12060793

Fahim, R. E., Kessler, P. D., \& Kalnik, M. W. (2013). Therapeutic vaccines against tobacco addiction. Expert Review of Vaccines, 12(3), 333-342. doi: 10.1586/erv.13.13

Farhangi, A., Akbarzadeh, A., Mehrabi, M. R., Chiani, M., Saffari, Z., Mortazavi, M., ... Ghassemi, S. (2012). Immunotherapy of 436 morphine addicts by therapeutic morphine vaccine in Kerman Province (I.R. Iran). Journal of Vaccines and Vaccinology, 3(6), 1-6. doi: 10.4172/2157-7560.1000154

Fraser, C. C., Altreuter, D. H., Ilyinskii, P., Pittet, L., LaMothe, R. A., Keegan, M., ... Kishimoto, T. K. (2014). Generation of a universal CD4 memory $T$ cell recall peptide effective in humans, mice and non-human primates. Vaccine, 32(24), 2896-2903. doi: 10.1016/j.vaccine.2014.02.024

Haney, M, Gunderson, E. W., Jiang, H, Collins, E. D., \& Foltin, R. W. (2010). Cocaine-specific anti-bodies blunt the subjective effects of smoked cocaine in humans. Biological Psychiatry, 67(1), 5965. doi: /10.1016/j.biopsych.2009.08.031

Hatsukami, D. K., Jorenby, D. E., González, D., Rigotti, N. A., Glover, E. D., Oncken, C. A, ... Rennard, S. I. (2011). Immunogenicity and smoking-cessation outcomes for a novel nicotine immunotherapeutic. Clinical Pharmacology \& Therapeutics, 89(3), 392399. doi: 10.1038/clpt.2010.317

Hatsukami, D. K., Rennard, S., Jorenby, D., Fiore, M., Koopmeiners, J., de Vos, A., ... Kalnik, M. W. (2005). Safety and immunogenicity of a nicotine conjugate vaccine in current smokers. Clinical Pharmacology \& Therapeutics, 78(5), 456-467. doi: 10.1016/ jclpt.2005.007

Hatsukami, D. K., Stead, L. F., \& Gupta, P. C. (2008). Tobacco addiction. Lancet, 371(9629), 2027-2038. doi: 10.1016/s01406736(08)60871-5

Havermans, A., Vuurman, E. F., van den Hurk, J., Hoogsteder, P., \& van Schayck, O. C. P. (2014). Treatment with a nicotine vaccine does not lead to changes in brain activity during smoking cue exposure or a working memory task. Addiction, 109(8), 12601267. doi:10.1111/add. 12577 
Hicks, M. J., De, B. P., Rosenberg, J. B., Davidson, J. T., Moreno, A. Y., Janda K. D., ... Crystal, R. G. (2011). Cocaine analog coupled to disrupted adenovirus: a vaccine strategy to evoke high-titer immunity against addictive drugs. Molecular Therapy, 19(3), 612-619. doi: 10.1038/mt.2010.280

Hoogsteder, P. H. J., Kotz, D., van Spiegel, P. I., Viechtbauer, W., \& van Schayck, O. C. (2014). Efficacyof the nicotine vaccine 3'-AmNic-rEPA (NicVAX) co-administered with varenicline and counselling for smoking cessation: a randomized placebo-controlled trial. Addiction, 109(8), 1252-1259. doi: 10.1111/add.12573

Kinsey, B. (2014). Vaccines against drugs of abuse: where are we now? Therapeutic Advances in Vaccines, 2(4), 106-117. doi: $10.1177 / 2051013614537818$

Kinsey, B. M., Jackson, D. C., \& Orson, F. M. (2009). Anti-drug vaccines to treat substance abuse. Immunology and Cell Biology, 87(4), 309-314. doi:10.1038/icb.2009.17

Kosten, T. R., Domingo, C. B., Shorter, D., Orson, F., Green, C., Somoza, E., ... Kampman, K. (2014). Vaccine for cocaine dependence: a randomized double-blind placebo-controlled efficacy trial. Drug and Alcohol Dependence, 140, 42-47. doi: 10.1016/j. drugalcdep.2014.04.003

Kosten, T., \& Owens, S. M. (2005). Immunotherapy for the treatment of drug abuse. Pharmacology \& Therapeutics, 108(1), 76-85. doi:10.1016/j.pharmthera.2005.06.009

Kosten, T. A., Shen, X. Y., O'Malley, P. W., Kinsey, B. M., Lykissa, E. D., Orson, F. M., ... Kosten, T. R. (2013). A morphine conjugate vaccine attenuates the behavioral effects of morphine in rats. Progress in Neuro-Psychopharmacology \& Biological Psychiatry, 45, 223-229. doi: 10.1016/j.pnpbp.2013.05.012

Li, Q. Q., Luo, Y. X., Sun, C. Y., Xue, Y. X., Zhu, W. L., Shi, H. S., ... Lu, L. (2011). A morphine/heroin vaccine with new hapten design attenuates behavioral effects in rats. Journal of Neurochemistry, 119(6), 1271-1281. doi: 10.1111/j.1471-4159.2011.07502.x

Li, Q. Q., Sun, C. Y., Luo, Y. X., Xue, Y. X., Meng, S. Q., Xu, L. Z., ... Sun, H. Q. (2015). A conjugate vaccine attenuates morphine-and heroin-induced behavior in rats. The International Journal of Neuropsychopharmacology, 18(5), 1-11. doi: 10.1093/ ijnp/pyu093

Maoz, A., Hicks, M. J., Vallabhjosula, S., Synan, M., Kothari, P. J., Dyke, J. P., ... Crystal, R. G. (2013). Adenovirus capsid-based anti-cocaine vaccine prevents cocaine from binding to the nonhuman primate CNS dopamine transporter. Neuropsychopharmacology, 38(11), 2170-2178. doi:10.1038/npp.2013.114

Martell, B. A., Mitchell, E., Poling, J., Gonsai, K., \& Kosten, T. R. (2005). Vaccine pharmacotherapy for the treatment of cocaine dependence. Biological Psychiatry, 58(2), 158-164. doi: 10.1016/j. biopsych.2005.04.032

Martell, B. A., Orson, F. M., Poling, J., Mitchell, E., Rossen, R. D., Gardner, T., ... Kosten, T. R. (2009). Cocaine vaccine for the treatment of cocaine dependence in methadone-maintained patients: a randomized, double-blind, placebo-controlled efficacy trial. Archives of General Psychiatry, 66(10), 1116-1123. doi: 10.1001/archgenpsychiatry.2009.128
Maurer, P., Jennings, G. T., Willers, J., Rohner, F., Lindman, Y., Roubicek, K., ... Bachmann, M. F. (2005). A therapeutic vaccine for nicotine dependence: preclinical efficacy, and Phase I safety and immunogenicity. European Journal of Immunology, 35(7), 2031-2040. doi: 10.1002/eji.200526285

Miller, M. L., Aarde, S. M., Moreno, A. Y., Creehan, K. M., Janda, K. D., \& Taffe, M. A. (2015). Effects of active anti-methamphetamine vaccination on intravenous self-administration in rats. Drug Alcohol Dependence, 153, 29-36. doi: 10.1016/j.drugalcdep.2015.06.014

Miller, M. L., Moreno, A. Y., Aarde, M., Creehan, K. M., Vandewater, S. A., Vaillancourt, B. D., ... Taffe, M. A. (2013). A methamphetamine vaccine attenuates methamphetamine-induced disruptions in thermoregulation and activity in rats. Biological Psychiatry, 73(8), 721-728. doi: 10.1016/j.biopsych.2012.09.010

Moher, D., Liberati, A., Tetzlaff, J., \& Altman D. G. (2009). Preferred reporting items for systematic reviews and meta-analyses: the PRISMA statement. BMJ, 339, b2535. doi: 10.1136/bmj.b2535

Montoya, I. D. (2012). Advances in the development of biologics to treat drug addictions and overdose. Adicciones, 24(2), 95-103. doi: 10.20882/adicciones. 101

Montoya, I. D. (Ed.). (2016). Biologics to treat substance use disorders. Switzerland: Springer International Publishing. doi: 10.1007/978-3-319-23150-1

Moreno, A. Y., \& Janda, K. D. (2009). Immunopharmacotherapy: vaccination strategies as a treatment for drug abuse and dependence. Pharmacology Biochemistry and Behavior, 92(2), 199205. doi:10.1016/j.pbb.2009.01.015

Moreno, A. Y., Mayorov, A. V., \& Janda, K. D. (2011). Impact of distinct chemical structures for the development of a methamphetamine vaccine. Journal of American Chemical Society, 133(17), 6587-6595. doi: 10.1021/ja108807j

Ohia-Nwoko, O., Kosten, T. A., \& Haile, C. N. (2016). Animal models and the development of vaccines to treat substance use disorders international review of neurobiology. International Review of Neurobiology, 126, 263-291. doi: 10.1016/bs.irn.2016.02.009

Orson, F. M., Rossen, R. D., Shen, X., López, A. Y., Wu, Y., \& Kosten, T. R. (2013). Spontaneous development of IGM anti-cocaine antibodies in habitual cocaine users: effect on IGG antibody responses to a cocaine cholera toxin b conjugate vaccine. The American Journal on Addictions, 22(2), 169-174. doi: 10.1111/j.1521. 0391.2013.00314.x

Orson, F. M., Wang, R., Brimijoin, S., Kinsey, B. M., Singh, R. A. K., Ramakrishnan, M., ... Kosten, T. R. (2014). The future potential for cocaine vaccines. Expert Opinion on Biological Therapy, 14(9), 1271-1283. doi: 10.1517/14712598.2014.920319

Raleigh, M. D., Pentel, P. R., \& LeSage, M. G. (2014). Pharmacokinetic correlates of the effects of a heroin vaccine on heroin self-administration in rats. PLoS One, 9(12), e115696. doi 10.1371/journal.pone.0115696

Rüedi-Bettschen, D., Wood, S. L., Gunnell, M. G., West, C. M., Pidaparthi, R. R., Carroll F. I., ... Owens, S. M. (2013). Vaccination protects rats from methamphetamine-induced impairment of be- 
havioral responding for food. Vaccine, 31(141), 4596-4602. doi: 10.1016/j.vaccine.2013.07.038

Salazar-Juárez, A., Barbosa-Méndez, S., Jurado, N., Hernández-Miramontes, R., Leff, P., \& Antón, B. (2016). Mirtazapine prevents induction and expression of cocaine-induced behavioral sensitization in rats. Progress in Neuro-Psychopharmacology and Biological Psychiatry, 68, 15-24. doi: 10.1016/j.pnpbp.2016.02.010

Schlosburg, J. E., Vendruscolo, L. F., Bremer, P. T., Lockner, J. W., Wade, C. L., Nunes, A. A. K.,... Koob, G. F. (2013). Dynamic vaccine blocks relapse to compulsive intake of heroin. Proceedings of the National Academy of Sciences of the United States of America, 110(22), 9036-9041. doi: 10.1073/pnas.1219159110

Shen, X. Y., Kosten, T. A., López, A. Y., Kinsey, B. M., Kosten, T. R., \& Orson, F. M. (2013). A vaccine against methamphetamine attenuates its behavioral effects in mice. Drug Alcohol Dependence, 129(1-2), 41-48. doi: 10.1016/j.drugalcdep.2012.09.007

Shen, X., Orson, F. M., \& Kosten, T. R. (2011). Anti-addiction vaccines. F1000 Medicine Reports, 3, 20. doi: 10.3410/M3-20

Shorter, D., \& Kosten, T. R. (2011). Novel pharmacotherapeutic treatments for cocaine addiction. BMC Medicine, 9(119). doi: 10.1186/1741-7015-9-119

Stowe, G. N., Schlosburg, J. E., Vendruscolo, L. F., Edwards, S., Misra, K. K., Schulteis, G., ... Janda K. D. (2011). Developing a vaccine against multiple psychoactive targets: a case study of heroin. Central Nervous System \& Neurological Disorders Drug Targets, 1O(8), 865-875. doi: 10.2174/187152711799219316

Stowe, G. N., Vendruscolo, L. F., Edwards, S., Schlosburg, J. E., Misra, K. K., Schulteis, G., ... Janda, K. D. (2011). A vaccine strategy that induces protective immunity against heroin. Journal of Medicinal Chemistry, 54(14), 5195-5204. doi: 10.1021/ jm200461m
Tonstad, S., Heggen, E., Giljam, H., Lagerbäck, P. Å., Tønnesen, P., Wikingsson, L.D., ... Fagerström, K. O. (2013). Niccine®, a nicotine vaccine, for relapse prevention: a phase II, randomized, placebo-controlled, multicenter clinical trial. Nicotine \& Tobacco Research, 15(9), 1492-1501. doi: 10.1093/ntr/ntt003

Torres, O. B., Jalah, R., Rice, K. C., Li, F., Antoline, J.F., Iyer, M. R., ... Matyas, G. R. (2014). Characterization and optimization of heroin hapten-BSA conjugates: method development for the synthesis of reproducible hapten-based vaccines. Analytical and Bioanalytical Chemistry, 406(24), 5927-5937. doi: 10.1007/ s00216-014-8035-x

Veilleux, J. C., Colvin, P. J., Anderson, J., York, C., \& Heinz, A. J. (2010). A review of opioid dependence treatment: pharmacological and psychosocial interventions to treat opioid addiction. Clinical Psychology Review, 30(2), 155-166. doi:10.1016/j.cpr. 2009.10.006

Wagena, E. J., de Vos, A., Horwith, G., \& van Schayck, C. P. (2008). The immunogenicity and safety of a nicotine vaccine in smokers and nonsmokers: results of a randomized, placebo-controlled phase 1/2 trial. Nicotine \& Tobacco Research, 10(1), 213-218. doi: 10.1080/14622200701704921

Wee, S., Hicks, M. J., De, B. P., Rosenberg, J. B., Moreno, A. Y., Kaminsky, S. M., ... Koob, G. F. (2012). Novel cocaine vaccine linked to a disrupted adenovirus gene transfer vector blocks cocaine psychostimulant and reinforcing effects. Neuropsychopharmacology, 37(5), 1083-1091. doi: 10.1038/npp.2011.200

Zalewska-Kaszubska, J. (2015). Is immunotherapy an opportunity for effective treatment of drug addiction? Vaccine, 33(48), 65456551. doi: 10.1016/j.vaccine.2015.09.079 